\title{
Title Study of Prevalence of Hypertension and Knowledge, Attitude and Behavior Toward Hypertension of Prison Inmates in Jinan
}

\author{
Dongying Sun, Jian Wang² and Anli Leng, ${ }^{2, a, *}$ \\ ${ }^{1}$ Shandong Police Hospital, Jinan, Shandong, China \\ ${ }^{2}$ School of Public Health, Shandong University, Jinan, Shandong, China \\ aanlijiayou@163.com \\ *corresponding author
}

Keywords: Hypertension, Knowledge-Attitude-Behavior, Quality of Life

\begin{abstract}
Objective To survey the prevalence of hypertension and knowledge,attitude and behavior towards hypertension of prison inmates in Jinan and provide scientific basis for strategies and methods in comprehensive prevention and control, health education and health promotion. Methods All detainees in Jinan City were chosen by cluster random sampling.A self-designed interview questionnaire was used. Data collected were analyzed by stata12.0. Results There were 192 effective questionnaire in total. And the prevalence rate of hypertension was $30.73 \%$. Significant difference was observed between different genders, but no significant difference was observed between different ages which had the highest prevalence rate in the group aged between 29 and 48. As for the education background, higher prevalence rate was in group with lower education. The rate of regular drug-taking was lower in hypertensive patients. The awareness rate of hypertension and the awareness rate of hypertension complications were both lower in patients than that in other group. The unhealthy behavior rates were high not in the patients but also in the other group. The mean scores of the quality of life of the prison inmates was 75.12. Conclusion The prevalence of hypertension of prison inmates in Jinan City was higher than that of the average level in Jinan City, and the knowledge, attitude and behavior towards hypertension of detainees was lower. It is urgent to strengthen the health education of hypertension prevention,combined with living habits and custom in the prison.
\end{abstract}

\section{济南某监狱在押人员高血压患病情况及其知信行调查}

\author{
孙冬英 ${ }^{1}$, 王健 ${ }^{2}$, 冷安丽 ${ }^{2, a, *}$ \\ ${ }^{1}$ 山东省警官总医院, 济南, 山东, 中国
}

${ }^{2}$ 山东大学公共卫生学院社会医学与卫生事业管理学和卫生经济实验与公共政策研究中心, 济南, 山东,

$$
\begin{gathered}
\text { 中国 } \\
\text { aanlijiayou@163.com } \\
\text { *通讯作者 }
\end{gathered}
$$

关键词: 高血压, 知信行, 生命质量

摘要: 目的了解济南市某监狱在押人员高血压患病现状及其知信行实际情况, 为开展高血压 综合防控和健康教育与健康促进策略提供科学依据。方法 按整体抽样（整群随机抽样法）的 方法对某监狱的全部在押人员进行问卷调查, 调查数据经 stata 12.0 软件进行统计学分析。结果 
共收集有效问卷 192 份, 高血压的患病率为 $30.73 \%$, 男女患病差异有统计学意义，不同年龄 高血压患病率差异有统计学意义, 患病率在 29-48 岁年龄段最高; 文化程度低者患病率高于文 化程度高者。既往诊断为高血压病患者遵医嘱规律服药率较低。高血压组高血压知识知晓率普 遍低于非高血压组, 且对高血压并发症的认知程度普遍较低; 高血压组和非高血压组对高血压 相关不健康行为形成率处于较高水平，且两者差异不显著。在监狱在押人员的生命质量平均得 分为 75.12。结论 济南市某监狱在押人员的高血压患病率高于济南市的一般水平, 高血压相关 知信行水平较低, 应结合监狱生活习惯进一步加强高血压防治健康教育和健康促进工作。

\section{中图分类号：R 186 文献标志码：A}

\section{1. 引言}

高血压是主要慢性疾病之一, 发病率高、致残率高、死亡率高[1],同时, 高血压会增加心 脏病发作、脑高血压是主要慢性疾病之一，发病率高、致残率高、死亡率高[1], 同时，高血 压会增加心脏病发作、脑卒中和肾衰竭等风险, 严重影响患者的生活质量[2]。研究发现患者 的膳食行为和生活方式对高血压的产生和发展有非常重要的影响[1]。已有理论证明，良好的 知识可以促进正确的信念，并形成健康的行为[3],是高血压预防控制的有效途径。监狱在押人 员作为特殊的群体, 目前关于其高血压相关研究比较少。本研究旨在通过了解济南市某监狱在 押人员高血压患病现状及其知、信、行实际情况，为有效控制高血压，进一步开展高血压健康 教育与健康促进策略提供科学依据。

\section{2. 研究对象}

2.1 对象的选择 2016 年 4 月至 2016 年 5 月, 采用整体抽样方法调查济南某监狱在押人员的高 血压患病情况, 以及高血压相关知、信、行。调查对象接受访问前, 由调查员详细告知其本次 研究的相关事项, 并告知其有权拒绝回答本次访问中的任一问题, 经调查对象知情同意后展开 调查。

\section{3. 调查方法}

\section{1 测量工具}

采用自行设计的结构式调查问卷，问卷内容主要包括社会人口学特征，高血压相关知、信、 行知识，高血压患者遵医嘱情况调查表和欧洲五维健康量表 EQ-5D 等。

\section{2 资料收集方法}

采用自行设计的结构式调查问卷，由经过培训的监狱护理人员发放调查问卷，采用统一的 指导语。若调查对象不识字，则一律由研究人员口述问题和选项，并代填。以面对面询问的方 式进行入户调查来收集数据。共发放调查问卷 198 份，回收有效问卷 192 份，有效回收率为 $96.97 \%$ 。

\section{3 统计学方法}

使用 Data Easy 3.3 软件建立数据库, 数据采用双次录入确保质量可靠。采用 SPSS 20 软件 进行统计分析。一般资料采用统计描述、组间和组内构成比的分析采用卡方检验。以 $\mathrm{P}<0.05$ 为差异具有统计学意义。

\section{4. 结果}

\section{1 调查对象的基本特征}

被调查人群中男性 160 人,女性 32 人, 男女性别比为 $5: 1$ 。年龄在 19 岁至 73 岁之间, 29 38 岁所占比重最大，占 $40.10 \%$ 。48.96\%拥有小学初中文化程度，33.85\%和 $9.90 \%$ 的 BMI 指数显 
示为偏胖或肥胖。仅有 $44.27 \%$ 两周前量过血压。已诊断高血压患者共 59 人，占被调查人群的 $30.73 \%$ 。详见表 1

表 1 研究对象的基本特征

\begin{tabular}{|c|c|c|c|c|c|}
\hline 基本特征 & 人数 & 构成比（\%) & 基本特征 & 人数 & $\begin{array}{c}\text { 构成比 } \\
(\%)\end{array}$ \\
\hline 性别 & & & 大专及以上 & 39 & 20.31 \\
\hline 男 & 160 & 83.33 & BMI & & \\
\hline 女 & 32 & 16.67 & 偏瘦（<18.5） & 9 & 4.69 \\
\hline 年龄（岁） & & & 正常（18.5-23.9） & 99 & 51.56 \\
\hline $19 \sim 28$ & 31 & 16.15 & 偏胖（24～27.9） & 65 & 33.85 \\
\hline $29 \sim 38$ & 77 & 40.1 & 肥胖 $(\geqslant 28)$ & 19 & 9.9 \\
\hline $39 \sim 48$ & 51 & 26.56 & 最近一次血压测量 & & \\
\hline $49 \sim 58$ & 23 & 11.98 & 两周前 & 85 & 44.27 \\
\hline $59 \sim$ & 10 & 5.21 & 两周到四周前 & 46 & 23.96 \\
\hline 受教育程度 & & & 大于四周前 & 61 & 31.77 \\
\hline 文盲 & 8 & 4.17 & 是否确诊患有高血压 & & \\
\hline 小学、初中 & 94 & 48.96 & 是 & 59 & 30.73 \\
\hline 高中 & 51 & 26.56 & 否 & 133 & 69.27 \\
\hline
\end{tabular}

\section{2 既往诊断为高血压病患者遵医嘱情况}

既往诊断为高血压病患者遵医嘱规律服药率较低。192 人中，既往诊断为高血压病患者 59 人, 其中有 24 人正在服用降压药，服药率为 $43.64 \%$, 其中 $73.68 \%$ 人遵医嘱有规律服药。关 于既往诊断高血压病患者不遵医嘱规律服药的原因, 忘记和自觉症状改善是可以停药所占比例 最高，分别占 $44.68 \%$ 和 $36.17 \%$ （见表 2 ）

表 2 既往诊断为高血压患者不能规律服药的原因分析（n,\%）

\begin{tabular}{ccc}
\hline 原因 & $\mathrm{n}$ & 构成比 $(\%)$ \\
\hline 经济原因 & 7 & 14.89 \\
忘记 & 21 & 44.68 \\
药物不良反应 & 13 & 27.66 \\
服用不方便 & 8 & 17.02 \\
自觉症状改善是可以停药 & 17 & 36.17 \\
没有时间就诊 & 5 & 10.64 \\
\hline
\end{tabular}

\section{3 不同人口学特征下高血压患病情况}

男女患病率差异有统计学意义，不同年龄高血压患病率差异有统计学意义，在 39-48 年龄 段的人群患病率最高, 达 $41.18 \%$; 文化程度低者患病率高于文化程度高者, 文盲的患病率为 $50 \%$, 小学的患病率为 $30.80 \%$, 大专及以上学历的患病率较低, 不同文化程度高血压患病率 差异无统计学意义。研究发现, BMI 值越高, 高血压的患病率就越高, 二者具有统计学意义。 
表 3 不同特征的调查对象的高血压患病情况

\begin{tabular}{|c|c|c|c|c|c|c|}
\hline 人口学特征 & \multicolumn{2}{|c|}{ 高血压组(n=59) } & \multicolumn{2}{|c|}{ 非高血压组 $(n=133)$} & 卡方值 & $\mathrm{P}$ 值 \\
\hline & 人数 & 百分比 $(\%)$ & 人数 & 百分比 $(\%)$ & & \\
\hline 性别 & & & & & 5.99 & 0.01 \\
\hline 男 & 55 & 93.22 & 105 & 78.95 & 65.62 & \\
\hline 女 & 4 & 6.78 & 28 & 21.05 & & \\
\hline 年龄（岁） & & & & & 9.60 & 0.05 \\
\hline $19 \sim 28$ & 4 & 6.78 & 27 & 20.30 & & \\
\hline $29 \sim 38$ & 21 & 35.59 & 56 & 42.10 & & \\
\hline $39 \sim 48$ & 21 & 35.59 & 30 & 22.56 & & \\
\hline $49 \sim 58$ & 8 & 13.56 & 15 & 11.28 & & \\
\hline $59 \sim$ & 5 & 8.48 & 5 & 3.76 & & \\
\hline 受教育程度 & & & & & 3.81 & 0.28 \\
\hline 文盲 & 4 & 6.78 & 4 & 3.01 & & \\
\hline 小学、初中 & 29 & 49.15 & 65 & 48.87 & & \\
\hline 高中 & 18 & 30.51 & 33 & 24.81 & & \\
\hline 大专及以上 & 8 & 13.56 & 31 & 23.31 & & \\
\hline BMI & & & & & 12.60 & 0 \\
\hline 偏瘦（<18.5） & 2 & 3.39 & 7 & 5.26 & & \\
\hline 正常（18.5-23.9） & 23 & 38.98 & 76 & 57.14 & & \\
\hline 偏胖（24～27.9） & 22 & 37.29 & 43 & 32.33 & & \\
\hline 肥胖 $(\geqslant 28)$ & 12 & 20.34 & 7 & 5.27 & & \\
\hline
\end{tabular}

\section{4 不同血压水平下的某监狱在押人员对高血压相关知识知晓情况}

高血压组对高血压知识知晓率普遍低于非高血压组, 且对高血压并发症的认知程度普遍较 低; 高血压组和非高血压组相比, 除肥胖、过量饮酒、缺乏运动, 脑卒中等两组差异均有统计 学意义外, 其它均无统计学意义。 
表 4 高血压患者与非高血压患者相关知识的知晓情况

调查项目

高血压组

非高血压组

卡方检验

$\mathrm{P}$ 值

\begin{tabular}{ccccccc}
\hline & 人数 & 知晓率 $(\%)$ & 人数 & 知晓率 $(\%)$ & & \\
高血压的诊断标准 & 38 & 64.41 & 86 & 64.66 & 0.00 & 0.97 \\
高血压的危险因素 & & & & & & \\
肥胖 & 45 & 76.27 & 120 & 90.23 & 6.59 & 0.01 \\
精神紧张 & 43 & 72.88 & 106 & 79.70 & 1.09 & 0.30 \\
高盐饮食、高脂饮食 & 43 & 72.88 & 107 & 80.45 & 1.37 & 0.24 \\
吸烟 & 48 & 80.36 & 119 & 89.47 & 2.38 & 0.12 \\
过量饮酒 & 48 & 81.36 & 123 & 92.48 & 5.19 & 0.02 \\
缺乏运动 & 48 & 81.36 & 125 & 93.98 & 7.31 & 0.00 \\
高血压并发症 & & & & & & \\
脑卒中 & 27 & 45.76 & 88 & 66.17 & 7.08 & 0.00 \\
心肌梗死 & 39 & 66.10 & 93 & 69.92 & 0.28 & 0.60 \\
肾病 & 10 & 16.95 & 19 & 14.29 & 0.23 & 0.63 \\
视网膜病变 & 17 & 28.81 & 52 & 39.10 & 1.88 & 0.17 \\
\hline
\end{tabular}

\section{5 不同血压水平下的某监狱在押人员相关行为}

高血压组和非高血压组对高血压相关不健康行为形成率处于较高水平。高血压组和非高血 压组相比, 除是否常吃油淢食物等两组差异有统计学意义外, 其它均无统计学意义。

表 5 不同血压人群对相关行为问题的回答情况

\begin{tabular}{|c|c|c|c|c|c|c|}
\hline 调查项目 & \multicolumn{2}{|c|}{ 患高血压组 } & \multicolumn{2}{|c|}{ 非高血压组 } & 卡方值 & $\mathrm{P}$ 值 \\
\hline & 人数 & $\begin{array}{l}\text { 肯定回答比 } \\
\text { 例 }(\%)\end{array}$ & 人数 & $\begin{array}{l}\text { 肯定回答比 } \\
\text { 例 }(\%)\end{array}$ & & \\
\hline 你两周内是否测过血压 & 25 & 42.37 & 60 & 45.11 & 0.12 & 0.72 \\
\hline $\begin{array}{c}\text { 你是否经常饮酒 } \\
\text { (每周饮酒 } 2 \text { 次以上) }\end{array}$ & 13 & 22.03 & 24 & 18.05 & 0.42 & 0.52 \\
\hline 你是否每天抽烟 & 27 & 45.76 & 50 & 37.59 & 1.14 & 0.29 \\
\hline $\begin{array}{c}\text { 你是否常吃腌制或熏制品 } \\
\text { (每周超过 } 3 \text { 次) }\end{array}$ & 13 & 22.03 & 30 & 22.56 & 0.01 & 0.94 \\
\hline $\begin{array}{c}\text { 你是否常吃新鲜蔬菜水果 } \\
\text { (每周超过 } 3 \text { 次) }\end{array}$ & 27 & 45.76 & 54 & 40.6 & 0.45 & 0.50 \\
\hline $\begin{array}{c}\text { 你是否常吃油淢食物 } \\
\text { (每周超过 } 3 \text { 次) }\end{array}$ & 11 & 18.64 & 11 & 8.27 & 4.33 & 0.04 \\
\hline 你是否规律运动 & 15 & 25.42 & 27 & 20.3 & 0.63 & 0.43 \\
\hline
\end{tabular}




\section{6 调查对象的生命质量情况}

使用 EQ-5D 量表测量调查对象的生命质量得分，分值为 0-100. 调查显示，在监狱在押人 员的生命质量平均得分为 75.12 , 其中高血压组的生命质量得分为 73.15 , 略低于非高血压组的 生命质量得分（75.99）。

\section{5. 讨论}

2013 年世界卫生组织将高血压定为主题，明确提高对高血压起因和后果的认识，提供如 何预防高血压及并发症的信息，同时鼓励成年人查血压，鼓励自我保护以有效预防高血压[2]。

本次调查结果显示，济南某监狱在押人员高血压患病率为 $30.73 \%$ ，高于济南市的一般水 平 $27.60 \%[4]$, 这可能与监狱在押人员的年龄结构和生活方式有关。因此，在高血压防治中， 应该因地制宜，结合监狱生活的特点开展高血压相关的健康教育和工作。

文化程度是影响居民对疾病知晓程度和自我保健意识的一个重要因素[5]。研究发现，高 血压患者的文化程度主要集中在初中及以下, 这与王建萍等在徐州开展的居民高血压相关知信 行研究的结果相一致[6]。

与以往研究结果[6]不同的是，我们研究发现：济南市某监狱在押人员中高血压组对高血 压知识的知晓率普遍低于非高血压组, 尤其是在高血压危险因素中的肥胖、过量饮酒和缺乏运 动, 高血压并发症中的脑卒中等方面, 二者的差异具有统计学意义。鉴于针对多个危险因素开 展综合社区干预是控制慢性病流行的有效措施[7], 我们应多开展关于高血压危险因素和并发 症相关知识的健康教育，提高人群尤其是高血压患者对高血压相关知识的知晓率。此外高血压 组中超过三分之二的患者对于高血压可能会引起肾病和视网膜突变的认识严重不足, 这与王建 萍等的研究[6]一致。可见，济南市某监狱在押人员的高血压知识有待于进一步提高。

高血压的发生于不健康的行为习惯和生活方式密切相关, 吸烟、饮酒、缺乏体育锻炼、高 盐高脂饮食、睡眠不足等不良生活习惯是造成高血压的重要危险因素 [8]。本次研究发现，无 论是高血压者还是和非高血压者, 其不定期测量血压、吸烟和无规律运动等高血压相关的不健 康行为的形成率处于较高水平，相反，在过量饮酒、经常食用腌制熏制食物和油淢食物等方面 的不健康行为的形成率则较低。这可能与在监狱的特殊情况有关，在押人员每日的食品和酒由 监狱统一提供，故在饮食结构上二者没有明显的区别，且易形成良好的饮食习惯。此外，研究 还发现是否常吃油淢食物对两组差异有统计学意义，在该监狱在押人员中 39 岁以上的人数占 $43.75 \%$, 这部分人群同时又是患高血压的高发人群，因此该监狱应更加合理的制定饮食结构， 少油淢，少腌制食物，同时倡导低盐饮食和低脂饮食的饮食习惯，尤其是加强对 39 岁以上人 群的宣传和教育。

以前研究显示，大部分患者不知高血压应采取终生服药，不能坚持服药，科学合理服药 [6,9]。我们的研究也发现忘记服药和和自觉症状改善是可以停药是既往诊断高血压病患者不遵 医嘱规律服药的两大重要原因。此外，调查对象中仅有 $20 \%$ 左右的人会坚持有规律的运动。这 提示我们应开展健康教育, 鼓励人们改变不良的生活方式, 提高自我保护意识和遵医行为。

总之，对在押人员而言，监狱卫生机构是高血压防治第一线。在目前形势下，首先，应采 取有效措施引导高血压病患者主动配合治疗，提高遵医嘱规律服药率，并定期复诊，从而有效 的控制并发症发生。其次，应在监狱中定期开展高血压相关医疗保健知识讲座，以提高高血压 防治知识水平，促使在押人员形成有利于预防控制高血压的信念并采取促进健康的行为。最后， 应加强高血压的定期篮查工作，较早发现高血压患者，并对其采取能有效控制血压水平行为干 预措施，预防和控制高血压的发生发展，从而提高其生命质量。

\section{基金项目}

挪威研究委员会资助项目(196400/S50)。 


\section{参考文献}

[1] 朱宣辑.537 例高血压患者的高血压防治知信行分析[D].吉林大学, 2014.

[2] 佚名.世界卫生日主题: 高血压 [J].中华护理学杂志,2013,48(4):310

[3] 杜文民,王永铭,陈斌艳, 等.社区高血压人群生活质量及知信行的调查 $[\mathrm{J}]$.药物流行病学杂 志,2001,10(4):191-193.

[4] 陈宝海. 济南地区健康体检人中高血压发病情况调查 [J]. 中国误诊学杂 志,2010,10(31):7801-7801.

[5] 王卓,江国虹,常改,等.天津市农村某示范社区 15 岁以上居民高血压患病情况及其危险因素 [J]. 职业与健康, 2009, 25(6):626-628.

[6] 王建萍,石红丽,王霞,等.徐州市居民高血压患病现况及知信行调查[J]. 中国卫生事业管 理,2011,28(12):954-956.

[7] 张磊,史中锋,迟阿鲁,等.居民高血压、糖尿病及知信行社区干预效果评价 [J].中国公共卫 生,2013,29(4):608-611.

[8] 陈霞,汪云,刘玲,等.安庆市农村地区高血压患者 KAP 及影响因素研究[J].中国卫生事业管 理,2009,26(12):845-847.

[9] 杨华杰, 周志衡,王家龔.中山市农村居民高血压防治知信行调查 [J]. 中国公共卫 生,2011,27(8):1036-1037. 